\title{
Perdas de cloreto de mepiquat no algodoeiro por chuva simulada
}

\author{
Gustavo Pavan Mateus(1), Eduardo do Valle Lima(1) e Ciro Antonio Rosolem ${ }^{(1)}$
}

(1)Universidade Estadual Paulista, Faculdade de Ciências Agronômicas, Dep. de Produção Vegetal - Agricultura, Caixa Postal 237, CEP 18603-970 Botucatu, SP. E-mail: gmateus@fca.unesp.br, rosolem@fca.unesp.br

\begin{abstract}
Resumo - No Brasil, a cotonicultura localiza-se em regiões onde o índice de precipitação pluvial é de aproximadamente $2.000 \mathrm{~mm}$ anuais. A aplicação de reguladores de crescimento em condições com esse índice faz com que o produto aplicado seja lavado antes de ser absorvido pelas plantas. $\mathrm{O}$ objetivo deste trabalho foi avaliar o efeito da lavagem do cloreto de mepiquat aplicado no algodoeiro, por chuvas simuladas em diferentes períodos após a aplicação do produto. $\mathrm{O}$ trabalho foi realizado em casa de vegetação, utilizando vasos de $12 \mathrm{~L}$, com três plantas da cultivar DeltaOpal. No aparecimento do primeiro botão floral, aplicou-se cloreto de mepiquat na dose de $12,5 \mathrm{~g} \mathrm{ha}^{-1}$ de i.a. Os tratamentos consistiram de duas lâminas de chuva, 10 e $20 \mathrm{~mm}, \mathrm{a}$ 0, 2, 4, 8, 16 e 32 horas após aplicação do produto e sem chuva, com quatro repetições. Uma chuva de $10 \mathrm{~mm}$ foi suficiente para lavar o produto das folhas independentemente do tempo de ocorrência após a aplicação do regulador. Se houver ocorrência de chuva $0,2,4,8,16$ e 32 horas após a aplicação do produto, a reposição deve ser de 131, 125, 120 , 110,93 e $70 \mathrm{~mL} \mathrm{ha}^{-1}$, respectivamente.
\end{abstract}

Termos para indexação: Gossypium hirsutum, regulador de crescimento, altura de plantas, precipitação.

\section{Mepiquat chloride loss by simulated rain}

\begin{abstract}
Brazilian cotton production is located in regions in which pluvial precipitation indexes are around $2,000 \mathrm{~mm}$ per year. Growth regulator application in conditions under such indices can make product to be washed before being absorbed by the plants. The objective of this research was to evaluate the effect of mepiquat chloride wash by simulated rain occurring at different times after the product application on cotton plants. The experiment was accomplished in greenhouse conditions, in pots of $12 \mathrm{~L}$ with three cotton plants cv. DeltaOpal. Mepiquat Chloride was sprayed at pin-head square, at $12.5 \mathrm{~g} \mathrm{ha}^{-1}$ a.i. Treatments consisted on two simulated rainfalls intensity (10 and $20 \mathrm{~mm}$ ) at $0,2,4,8,16$ and 32 hours after the product application and a treatment without rain, with four replications. A $10 \mathrm{~mm}$ rain was enough to wash the product from cotton leaves irrespectively of the time after application of the regulator. If there is rain $0,2,4,8,16$ or 32 hours after product application, it should be reapplied 131, 125, 120,110, 93 and $70 \mathrm{~mL} \mathrm{ha}^{-1}$, respectively.
\end{abstract}

Index terms: Gossypium hirsutum, plant growth regulator, plant height, rainfall.

\section{Introdução}

Nas áreas onde o algodoeiro (Gossypium hirsutum L.) é cultivado em solos corrigidos e condições climáticas favoráveis, freqüentemente, verifica-se crescimento vegetativo excessivo, resultando em plantas altas, com densa folhagem, o que impede a penetração da radiação solar, dificulta a aplicação de defensivos e pode inviabilizar a colheita mecanizada (Marur, 1998; Lamas, 2001). Nas pequenas propriedades agrícolas, o crescimento excessivo do algodoeiro muitas vezes é controlado por meio da capação, feita manualmente, planta por planta, eliminando-se a gema apical (Carvalho et al., 1994), mas o modo mais eficaz no controle do crescimento da planta é o uso de reguladores de crescimento.
Os reguladores de crescimento são substâncias químicas naturais ou sintéticas que podem ser aplicadas diretamente nos vegetais para alterar os processos vitais ou estruturais, por meio de modificações no balanço hormonal das plantas, com a finalidade de aumentar a produção, melhorar a qualidade ou facilitar a colheita (Laca-Buendia, 1989; Lamas, 2001). No mercado brasileiro, encontram-se três produtos comerciais recomendados como reguladores de crescimento em algodoeiro: Cycocel (cloreto de clorocolina), Tuval (cloreto de chlormequat) e Pix (cloreto de mepiquat) (Carvalho et al., 1994). Esses produtos têm mecanismos e modos de ação semelhantes, ou seja, interferem na biossíntese do ácido giberélico, inibindo-a, o que resulta em redução do crescimento, em razão da menor elongação celular (Lamas, 2001). 
O cloreto de mepiquat (cloreto 1,1-dimetil piperidíneo) é um composto orgânico, solúvel em água, que se apresenta como uma solução aquosa, incolor e inodora (Cruz et al., 1982). É um produto sistêmico, absorvido principalmente pelas partes verdes da planta, cuja $\mathrm{LD}_{50}$ é de $1.605 \mathrm{mg} \mathrm{kg}^{-1}$ de peso vivo (Reddy et al., 1995). De acordo com Reddy et al. (1996), esse regulador é translocado de forma ascendente e descendente, pelo xilema e floema, de maneira uniforme, e não é degradado nas plantas de algodão.

O efeito potencial da aplicação do regulador cloreto de mepiquat na cultura do algodoeiro é a melhoria da arquitetura das plantas, com modificações favoráveis do microclima da vegetação, o que reduz a incidência da podridão dos frutos e melhora a qualidade da fibra produzida (Marur, 1998). Barbosa \& Castro (1983) constataram aumentos na produção, em razão do equilíbrio entre altura da planta e índice de área foliar com número de ramos produtivos e de capulhos, que propiciaram maior translocação de carboidratos para os frutos. Outras modificações favoráveis são a redução do crescimento vegetativo, isto é, diminuição da altura, do tamanho dos ramos vegetativos e reprodutivos, do comprimento dos internódios e do número de folhas quando da colheita; o aumento da retenção de frutos nas primeiras posições dos ramos frutíferos; a abertura precoce dos frutos, com antecipação da colheita, que por sua vez pode contribuir para reduzir o ataque de pragas tardias; a maior uniformização do estande de cultivo, melhorando a eficiência dos tratos culturais e da colheita mecanizada (Athayde \& Lamas, 1999; Lamas et al., 1999; Lamas \& Athayde, 1999; Lamas, 2001).

Os efeitos do cloreto de mepiquat sobre o algodoeiro dependem da dose utilizada, da época e modos de aplicação, da cultivar empregada, da data de semeadura, da população de plantas e das condições ambientais que se seguem ao tratamento, como temperatura e precipitação pluvial (Laca-Buendia, 1989; Marur, 1998; Athayde \& Lamas, 1999). Assim, segundo a recomendação da Embrapa (1997), devem-se aplicar doses baixas e múltiplas, iniciando na fase de primeiro botão floral em desenvolvimento. A primeira aplicação deve ser realizada quando o primeiro botão floral da primeira posição alcançar 3 mm de diâmetro. Isso ocorre entre 6 e 10 dias, dependendo da cultivar e da região, após o início da formação do botão floral. Quando $50 \%$ das plantas tiverem atingido este estádio, deve-se efetuar a aplicação de $12,5 \mathrm{~g} \mathrm{ha}^{-1}$ do princípio ativo.
Com relação ao fator precipitação pluvial, ressaltase que em boa parte das regiões onde se encontram as maiores áreas de cultivo do algodão no Brasil, os índices estão próximos de $2.000 \mathrm{~mm}$ anuais. Além disso, a melhor época recomendada para aplicação dos reguladores de crescimento coincide com o período de maior concentração pluvial. Existe, portanto, grande risco de o produto ser lavado pela água das chuvas, sem ter sido totalmente absorvido pelas plantas. Assim, seria interessante a reaplicação da quantidade de produto perdida, para que este possa exercer de forma efetiva o efeito esperado no controle do crescimento excessivo das plantas de algodão.

O objetivo deste trabalho foi avaliar a lavagem do cloreto de mepiquat aplicado no algodoeiro por chuvas em diferentes tempos após a aplicação do produto.

\section{Material e Métodos}

O trabalho foi realizado em casa de vegetação na Faculdade de Ciências Agronômicas, Unesp, no Município de Botucatu, SP, utilizando-se vasos de plástico de $12 \mathrm{~L}$, preenchidos com Latossolo Vermelho distrófico típico, textura média (Embrapa, 1999), passado em peneira de $4 \mathrm{~mm}$. A análise química, $0-20 \mathrm{~cm}$ (Raij et al., 2001), mostrou os seguintes atributos: $\mathrm{pH}\left(\mathrm{CaCl}_{2}\right), 5,0$; MO, $26 \mathrm{~g} \mathrm{dm}^{-3}$; H+Al, $46 \mathrm{mmol}_{\mathrm{c}} \mathrm{dm}^{-3}$; $\mathrm{P}$ (resina), $20 \mathrm{mg} \mathrm{dm}^{-3} ; 4,6,35,9$ e 14,4 mmol $_{\mathrm{c}} \mathrm{dm}^{-3} \mathrm{de} \mathrm{K}^{+}, \mathrm{Ca}^{2+} \mathrm{e}$ $\mathrm{Mg}^{2+}$, respectivamente; CTC de 100,6 $\mathrm{mmol}_{\mathrm{c}} \mathrm{dm}^{-3} \mathrm{e}$ $\mathrm{V}$ de $55 \%$. O solo foi misturado homogeneamente com $150 \mathrm{mg} \mathrm{dm}^{-3}$ de $\mathrm{K}_{2} \mathrm{O}, 150 \mathrm{mg} \mathrm{dm}^{-3}$ de $_{2} \mathrm{O}_{5}, 3 \mathrm{mg} \mathrm{dm}^{-3}$ de $\mathrm{Zn}$ e $1 \mathrm{mg} \mathrm{dm}^{-3}$ de $\mathrm{B}$, nas formas de cloreto de potássio, fosfato monoamônio, sulfato de zinco e ácido bórico. Em seguida, os vasos foram completados e o solo umedecido até $80 \%$ da capacidade de campo. Foram então cobertos com lona de plástico preta, permanecendo em incubação por um período de 21 dias.

A seguir realizou-se a semeadura de cinco sementes de algodão, por vaso, da cultivar DeltaOPAL, cujas principais características são: altura de 150 a $170 \mathrm{~cm}$, florescimento de 52 a 60 dias, resistente ao acamamento e produtividade em torno de $5.250 \mathrm{~kg} \mathrm{ha}^{-1}$ (Maeda Deltapine Monsanto Algodão, 2001). As sementes foram previamente tratadas com fungicida à base de thiram,

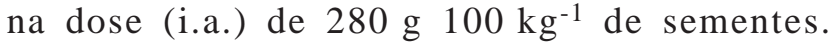
A emergência ocorreu cinco dias após a semeadura, sendo realizado o desbaste, deixando-se três plantas por vaso. Quando o terceiro nó vegetativo estava aparecendo, aplicaram-se, em cobertura, $50 \mathrm{mg} \mathrm{dm}^{-3} \mathrm{de} \mathrm{N}$, na forma de nitrato de amônio. 
Aos 38 dias após a emergência (DAE), ou seja, no aparecimento do primeiro botão floral, foi aplicado, em todas as plantas, o regulador de crescimento cloreto de mepiquat (cloreto 1,1-dimetil piperidíneo) (Reddy et al., 1990), na dose (i.a.) de 12,5 $\mathrm{g} \mathrm{ha}^{-1}$ e volume de aplicação de $150 \mathrm{~L} \mathrm{ha}^{-1}$ de calda, por meio de equipamento de simulação de pulverizador de barra. Simultaneamente, iniciaram-se os tratamentos.

O delineamento estatístico utilizado foi inteiramente casualizado, num esquema fatorial $2 \times 6+1$, ou seja, duas lâminas de chuva simulada ( 10 e $20 \mathrm{~mm}$ ), aplicadas em seis tempos $(0,2,4,8,16$ e 32 horas após aplicação do regulador de crescimento) e uma testemunha absoluta sem simulação de chuva, com quatro repetições.

$\mathrm{O}$ equipamento de simulação de pulverização e de chuva encontra-se instalado em sala fechada, constituindo-se de estrutura metálica com $3 \mathrm{~m}$ de altura por $2 \mathrm{~m}$ de largura, que permite o acoplamento de um carrinho suspenso a 2,5 $\mathrm{m}$ de altura. A esse carrinho acopla-se duas barras de pulverização; uma para a simulação de chuva e outra para pulverização de defensivos agrícolas, ambas com deslocamento horizontal de $6 \mathrm{~m}$. $\mathrm{O}$ equipamento encontra-se descrito detalhadamente em Silva (2000).

A organização das unidades experimentais ao longo do percurso das barras do simulador foram feitas de acordo com o espaçamento normalmente utilizado nas lavouras de algodão $(0,90 \mathrm{~m})$.

A barra de pulverização foi dotada com quatro bicos cônicos (Dx 8), com espaços de $0,50 \mathrm{~m}$ e posicionados a $0,50 \mathrm{~m}$ do dossel das plantas. $\mathrm{O}$ sistema foi operado à pressão de $35 \mathrm{kgf} \mathrm{cm}^{-2}$ e com velocidade de deslocamento de $1,0 \mathrm{~m} \mathrm{~s}^{-1}\left(3,6 \mathrm{~km} \mathrm{~h}^{-1}\right)$.

$\mathrm{Na}$ simulação de chuva, a barra foi fixada a $1,75 \mathrm{~m}$ de altura. Essa barra é dotada de três bicos de alta vazão (TK-SS-20), com espaços de 0,50 m. As gotas de chuva artificial foram geradas utilizando-se pressão de trabalho de $0,81 \mathrm{kgf} \mathrm{cm}^{-2}$, e velocidade de deslocamento de $0,0526 \mathrm{~m} \mathrm{~s}^{-1}$. Assim, a cada deslocamento total da barra, aplicaram-se 2,5 $\mathrm{mm}$ de chuva.

Imediatamente antes da aplicação do regulador de crescimento, efetuou-se a medição de altura de todas as plantas (colo até o último nó vegetativo). Após a aplicação dos tratamentos, cultivaram-se as plantas por 32 dias, realizando-se medições de altura em intervalos de 4 dias. Durante esse período, foram registradas, numa estação meteorológica portátil eletrônica, as temperaturas máxima e mínima do ar. No cálculo dos graus-dia acumulados, GDD $=[$ (temperatura máxima + temperatura mínima)/2] - temperatura base, utilizou-se $15^{\circ} \mathrm{C}$ como temperatura base.

Com as médias de altura para cada vaso, determinou-se o crescimento das plantas em função dos grausdia acumulados, ajustando-se as curvas por meio do modelo de Mitscherlich. Em seguida, calcularam-se as taxas de crescimento, derivando as equações ajustadas às curvas de crescimento. Estimou-se a atuação do produto em razão da ocorrência de chuvas em diferentes períodos após a sua aplicação, assim como calculou-se a dose a ser reposta com base na taxa de crescimento das plantas tomadas aos 230 graus-dia, período suficiente para o regulador de crescimento expressar seu efeito. A fórmula utilizada foi:

$\mathrm{R}=[1-(\mathrm{TCTe} / \mathrm{TCTt})] \times \mathrm{D}$

em que $\mathrm{R}$ é a dose a ser reposta, em $\mathrm{mL} \mathrm{ha}{ }^{-1}$; TCTe é a taxa de crescimento da testemunha, em $\mathrm{cm} \mathrm{GD}^{-1}$; TCTt é a taxa de crescimento dos tratamentos $(0,2,4$, 8, 16 e 32 horas), em $\mathrm{cm} \mathrm{GD}^{-1}$ e D é a dose utilizada do regulador de crescimento ( $\left.250 \mathrm{~mL} \mathrm{ha}^{-1}\right)$.

Ao final do experimento, as plantas foram cortadas rente ao solo, para determinação da massa de matéria seca e contagem do número de nós. Pela razão entre altura de plantas e número de nós, obteve-se a variável comprimento médio de internódios.

Os resultados obtidos foram submetidos à análise de variância, e as médias comparadas pelo teste de Tukey a 5\% de probabilidade. Quando apropriado, foram ajustadas curvas de regressão, escolhendo-se o modelo significativo de melhor ajuste.

\section{Resultados e Discussão}

As características das plantas de algodão, como altura final de plantas e comprimento médio de internódios, não foram afetadas pela interação dos fatores lâmina de chuva e tempo para aplicação, assim como não houve efeito isolado da lâmina de chuva em relação a essas variáveis (Tabela 1). Quanto ao fator isolado tempo para aplicação da chuva, constatou-se efeito significativo, sendo discutido separadamente. Não houve efeito significativo em relação às variáveis massa de matéria seca da parte aérea e número de nós.

O efeito não-significativo do fator lâminas de chuva em relação às variáveis estudadas pode ser atribuído à lavagem do produto com a menor quantidade de chuva aplicada $(10 \mathrm{~mm})$, que, provavelmente, foi suficiente 
para lixiviar quantidades significativas do produto. Devese ressaltar que se está discutindo quantidade de chuva e não intensidade (Rosolem et al., 2003), pois, por exemplo, a quantidade de $10 \mathrm{~mm}$, na realidade, corresponde a quatro pulsos de $2,5 \mathrm{~mm}$.

A maior altura final de plantas foi obtida no tratamento no qual a chuva foi simulada logo após aplicação do regulador de crescimento, sendo estatisticamente diferente da testemunha e do tratamento 32 horas após a aplicação do produto. Em contrapartida, chuvas simuladas 2, 4, 8 e 16 horas após aplicação do produto não se diferenciaram significativamente dos tratamentos que obtiveram a menor (32 horas e testemunha) e maior altura (0 hora) (Tabela 1).

Avaliando-se o comportamento de altura final (Tabela 1), por meio do crescimento das plantas após a aplicação dos tratamentos (Figura 1), confirma-se diminuição da altura das plantas com o atraso na aplicação da chuva. Este resultado pode ser atribuído ao maior intervalo de tempo, suficiente para que a superfície das folhas das plantas absorva o regulador de crescimento, acarretando, portanto, menor perda do produto por lavagem e, conseqüentemente, proporcionando redução da altura do algodoeiro.

Tabela 1. Altura, matéria seca final, número de nós e comprimento médio de internódio em razão de lâmina de chuva (L) e tempo para aplicação de chuva $(\mathrm{T})$, após pulverização de cloreto de mepiquat no algodoeiro ${ }^{(1)}$.

\begin{tabular}{ccccc}
\hline Tratamento & $\begin{array}{c}\text { Altura } \\
\text { final }(\mathrm{cm})\end{array}$ & $\begin{array}{c}\text { Matéria seca } \\
\text { final }(\mathrm{g} \text { planta }\end{array}$ & $\begin{array}{c}\text { Número } \\
\text { de nós }\end{array}$ & $\begin{array}{c}\text { Comprimento } \\
\text { médio de } \\
\text { internódios }(\mathrm{cm})\end{array}$ \\
\hline $\begin{array}{c}\text { Lâmina de chuva } \\
10 \mathrm{~mm}\end{array}$ & $77,1 \mathrm{a}$ & $32,2 \mathrm{a}$ & $6,4 \mathrm{a}$ & $6,6 \mathrm{a}$ \\
$20 \mathrm{~mm}$ & $77,0 \mathrm{a}$ & $30,4 \mathrm{a}$ & $6,6 \mathrm{a}$ & $6,6 \mathrm{a}$ \\
\hline Tempo (horas) & & & & \\
Testemunha ${ }^{(2)}$ & $64,7 \mathrm{~b}$ & $29,2 \mathrm{a}$ & $6,3 \mathrm{a}$ & $5,0 \mathrm{~b}$ \\
0 & $83,3 \mathrm{a}$ & $32,8 \mathrm{a}$ & $6,9 \mathrm{a}$ & $7,2 \mathrm{a}$ \\
2 & $74,2 \mathrm{ab}$ & $32,2 \mathrm{a}$ & $6,6 \mathrm{a}$ & $7,1 \mathrm{ab}$ \\
4 & $77,0 \mathrm{ab}$ & $32,4 \mathrm{a}$ & $6,6 \mathrm{a}$ & $6,8 \mathrm{ab}$ \\
8 & $75,1 \mathrm{ab}$ & $29,9 \mathrm{a}$ & $6,6 \mathrm{a}$ & $6,4 \mathrm{ab}$ \\
16 & $75,4 \mathrm{ab}$ & $32,1 \mathrm{a}$ & $6,4 \mathrm{a}$ & $6,4 \mathrm{ab}$ \\
32 & $70,7 \mathrm{~b}$ & $30,2 \mathrm{a}$ & $6,1 \mathrm{a}$ & $5,8 \mathrm{~b}$ \\
\hline Teste F & & & & \\
Lâmina & $0,01^{\mathrm{ns}}$ & $2,63^{\mathrm{ns}}$ & $0,77^{\mathrm{ns}}$ & $0,01^{\mathrm{ns}}$ \\
Tempo & $3,93^{* *}$ & $0,81^{\mathrm{ns}}$ & $1,11^{\mathrm{ns}}$ & $2,31^{\mathrm{n}}$ \\
L X T & $0,83^{\mathrm{ns}}$ & $0,88^{\mathrm{ns}}$ & $0,12^{\mathrm{ns}}$ & $0,40^{\mathrm{ns}}$ \\
\hline CV (\%) & 8,89 & 13,8 & 11,75 & 13,61 \\
\hline
\end{tabular}

${ }^{(1)}$ Médias seguidas da mesma letra não diferem entre si pelo teste de Tukey a 5\% de probabilidade. ${ }^{(2)}$ Testemunha absoluta sem simulação de

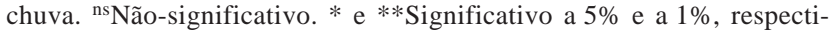
vamente, pelo teste $\mathrm{F}$.
Como a altura de plantas sofreu efeito significativo do fator tempo e a massa de matéria seca não diferiu entre os tratamentos, as plantas de menor porte, em contrapartida, apresentaram um ganho compensatório na massa de matéria seca em relação às de maior altura (Tabela 1). Esse comportamento pode ser justificado pelo fato de que, à medida que as plantas crescem, ocorre uma diminuição da razão de área foliar (Benincasa, 1988), ou seja, a aplicação do regulador proporcionou redução de altura, com a manutenção da massa de matéria seca, por causa de um possível espessamento das folhas. Barbosa \& Castro (1983) também verificaram a manutenção, praticamente constante, da massa de matéria seca de plantas de algodão tratadas com reguladores de crescimento, por causa da ampla variação do índice de área foliar. Outra hipótese seria o aumento da capacidade fotossintética nas plantas de algodão que tiveram menor lavagem do produto (Stuart et al., 1984), por causa do controle da concentração subestomática de $\mathrm{CO}_{2}$, ocasionando maiores taxas de assimilação de carbono (Marur, 1998).

Com relação ao número de nós, constata-se que não houve efeito significativo entre os tratamentos (Tabela 1). Já quanto ao comprimento médio de internódios, observou-se comportamento semelhante ao de altura de plantas (Tabela 1). Como o comprimento médio de internódios é o produto da divisão de altura de plantas pelo número de nós, a menor variação desta última justifica a maior participação da altura na determinação do comprimento de internódios. Assim, à medida que ocor-

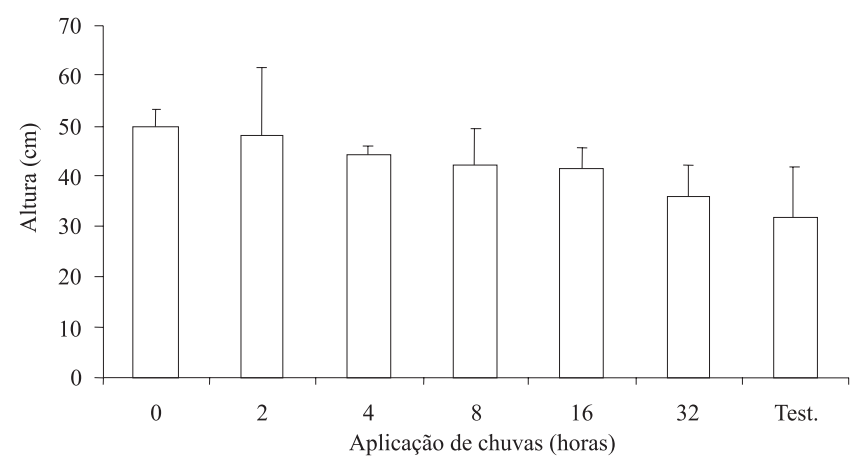

Figura 1. Altura de plantas de algodão, em cm, submetidas a diferentes tempos de aplicação de chuva após pulverização de cloreto de mepiquat. Barras em cada coluna representam a média \pm desvio-padrão. 
reu atraso na aplicação de chuva, constatou-se diminuição do comprimento médio de internódios, e para o tratamento chuva logo após a aplicação do produto (0 hora), as plantas atingiram $7,2 \mathrm{~cm}$ e, 32 horas depois, o valor chegou a somente $5,8 \mathrm{~cm}$. Isso evidencia o efeito da chuva na lavagem do produto. As plantas que tiveram tempo hábil para a absorção de quantidade suficiente do produto apresentaram menor comprimento de internódios, pois o regulador de crescimento atua na inibição da síntese de giberelinas, as quais exercem função na divisão celular e no alongamento de células (Reddy et al., 1992).

Aos 425 graus-dia acumulados (ao final do experimento), o máximo crescimento das plantas diferiu entre os tempos de simulação da chuva (Figura 2). Desta maneira, com o aumento do tempo para a aplicação da chuva, constatou-se diminuição do crescimento das plantas, isto é, o tratamento no qual a chuva foi simulada logo após a aplicação do produto (0 hora) apresentou um crescimento final maior do que nos demais tempos. Portanto, no tratamento 0 hora, verificou-se crescimento final de $83,6 \mathrm{~cm}$, ao passo que na testemunha (sem simulação de chuva) e na simulação de chuva 32 horas

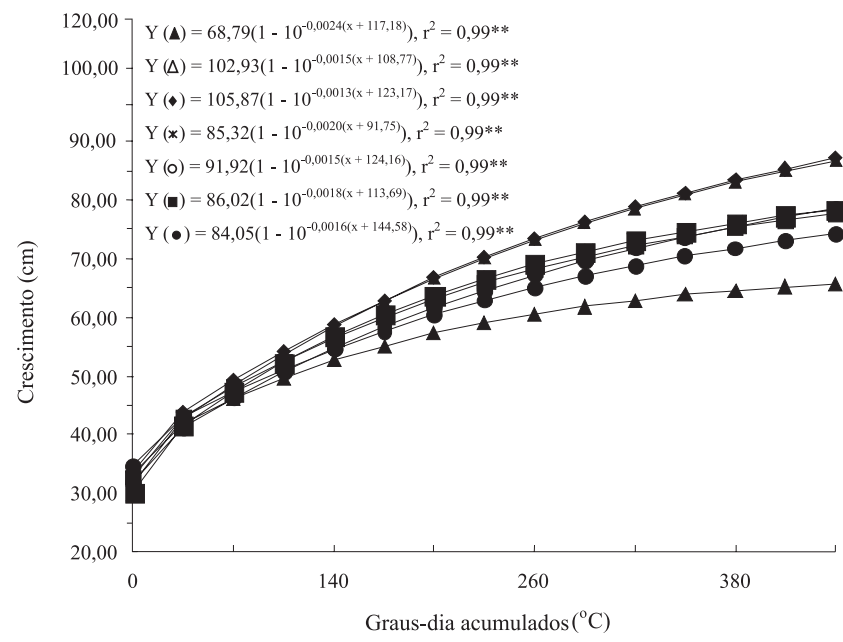

Figura 2. Relação entre o crescimento de plantas de algodão e os graus-dia acumulados descrita pelo modelo de Mitscherlich em razão da ocorrência de chuvas $0(\triangle), 2(\bullet)$, $4(\boldsymbol{*}), 8(\mathrm{O}), 16(\square)$ e 32 horas $(\bullet)$ após a pulverização de cloreto de mepiquat. Testemunha absoluta $(\boldsymbol{\Delta})$ sem simulação de chuva. $\mathrm{x}$ representa graus-dia acumulados $\left({ }^{\circ} \mathrm{C}\right)$ após aplicação dos tratamentos. **Significativo a $1 \%$ de probabilidade. após a aplicação do regulador de crescimento registraram-se apenas 64,7 e 70,7 cm, respectivamente. Este fato pode ser atribuído à maior lavagem do produto nos tratamentos em que a chuva foi simulada próximo à pulverização do regulador, não havendo tempo suficiente para sua absorção.

Independentemente das diferenças observadas entre os tratamentos, há uma tendência similar de comportamento (Figura 3). Inicialmente, ou seja, no período em que foi aplicado o regulador de crescimento (no aparecimento do ramo frutífero), a velocidade de crescimento é alta (aos 50 graus-dia), e a partir de então, os valores decrescem progressivamente, tendendo a uma estabilização a partir de 230 graus-dia. A diminuição da taxa de crescimento estimada em todos os tratamentos, com o avanço dos estádios fenológicos da planta, devese ao fato de que grande parte dos fotoassimilados são transportados para uso no desenvolvimento dos órgãos reprodutivos, que são drenos altamente competitivos (Souza \& Beltrão, 1999).

Com os resultados da taxa de crescimento, foi possível determinar a dose a ser reposta do regulador de crescimento em razão do tempo para ocorrência de chuva após a sua aplicação, ou seja, $0,2,4,8,16$ ou 32 horas após a aplicação do produto, a reposição necessária seria de $131,125,120,110,93$ e $70 \mathrm{~mL} \mathrm{ha}^{-1}$, respectivamente (Figura 4).

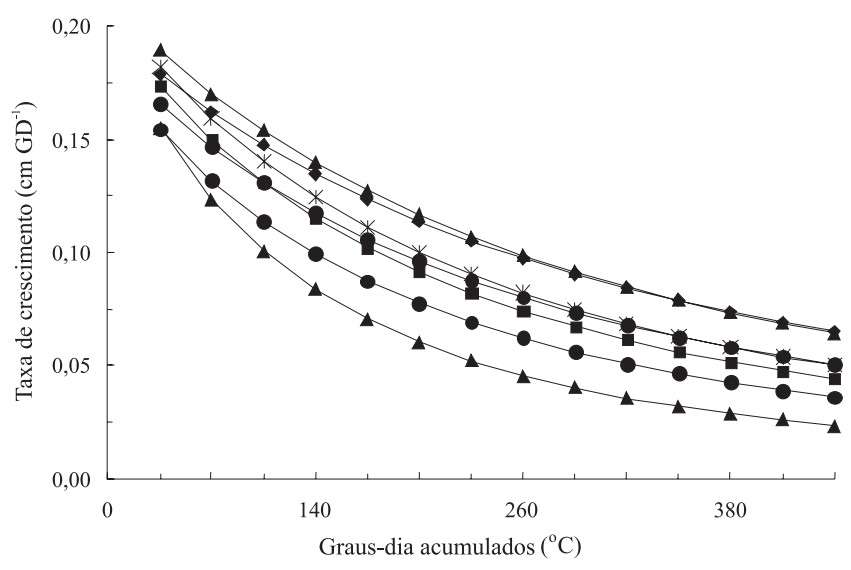

Figura 3. Relação entre a taxa de crescimento de plantas de algodão e os graus-dia acumulados em razão da ocorrência de chuvas $0(\triangle), 2(\bullet), 4(\boldsymbol{*}), 8(\bigcirc), 16(\square)$ e 32 horas $(\bullet)$ após a pulverização de cloreto de mepiquat. Testemunha absoluta (A) sem simulação de chuva. 


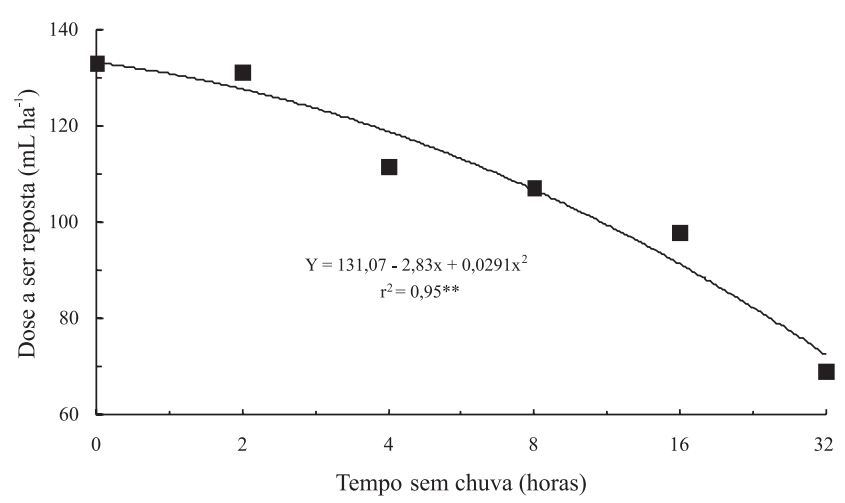

Figura 4. Dose de cloreto de mepiquat a ser aplicada na cultura de algodão em razão do tempo de ocorrência de chuva após a aplicação do produto (mL de produto com $50 \mathrm{~g} \mathrm{~L}^{-1}$ de i.a.).

\section{Conclusões}

1. Uma chuva de $10 \mathrm{~mm}$ é suficiente para a lavagem do regulador de crescimento.

2. Com a ocorrência de chuva até 16 horas após a aplicação do cloreto de mepiquat, faz-se necessário a reposição do produto.

\section{Referências}

ATHAYDE, M.L.F.; LAMAS, F.M. Aplicação seqüencial de cloreto de mepiquat em algodoeiro. Pesquisa Agropecuária Brasileira, v.34, p.369-375, 1999.

BARBOSA, L.M.; CASTRO, P.R.C. Desenvolvimento e produtividade de algodoeiros sob efeito de reguladores vegetais. Anais da Escola Superior de Agricultura Luiz de Queiroz, v.40, p.3386, 1983.

BENINCASA, M.M.P. Análise de crescimento de plantas: noções básicas. Jaboticabal: Funep, 1988. 42p.

CARVALHO, L.H.; CHIAVEGATO, E.J.; CIA, E.; KONDO, J.I.; SABINO, J.C.; PETTINELLI JÚNIOR, A.; BORTOLETTO, N.; GALLO, P.B. Fitorreguladores de crescimento e capação na cultura algodoeira. Bragantia, v.53, p.247-254, 1994.

CRUZ, L.S.F.; SABINO, N.P.; TOLEDO, N.M.P. Efeitos do cloreto de mepiquat empregado como fitorregulador sobre algodoeiro herbáceo (Gossypium hirsutum L. "IAC 16"). Planta Daninha, v.1, p.15-22, 1982.

EMBRAPA. Centro Nacional de Pesquisa de Algodão (Campina Grande, PB). Aplicação do regulador cloreto de mepiquat no crescimento e na produtividade do algodão herbáceo. Campina Grande, 1997. 71p.

EMBRAPA. Centro Nacional de Pesquisa de Solos (Rio de Janeiro, RJ). Sistema brasileiro de classificação de solos. Brasília: Embrapa-SPI; Embrapa-CNPS, 1999. 412p.
LACA-BUENDIA, J.P. Efeito de doses de reguladores de crescimento no algodoeiro (Gossypium hirsutum L.). Revista Brasileira de Fisiologia Vegetal, v.1, p.109-113, 1989.

LAMAS, F.M. Estudo comparativo entre cloreto de mepiquat e cloreto de chlormequat aplicados no algodoeiro. Pesquisa Agropecuária Brasileira, v.36, p.265-272, 2001.

LAMAS, F.M.; ATHAYDE, M.L.F. Efeito do cloreto de mepiquat e do thidiazuron sobre algumas características das sementes do algodoeiro. Pesquisa Agropecuária Brasileira, v.34, p.2015-2019, 1999.

LAMAS, F.M.; ATHAYDE, M.L.F.; BANZATTO, D.A.; FORTUNA, P.A. Cloreto de mepiquat, thidiazuron e ethephon aplicados no algodoeiro em Ponta Porã, MS. Pesquisa Agropecuária Brasileira, v.34, p.1871-1880, 1999.

MAEDA DELTAPINE MONSANTO ALGODÃO. Guia técnico DeltaOPAL. Uberlândia, 2001. 10p.

MARUR, C.J. Fotossíntese e translocação de carboidratos em algodoeiro submetidos à déficit hídrico após a aplicação de cloreto de mepiquat. Revista Brasileira de Fisiologia Vegetal, v.10, p.5964, 1998.

RAIJ, B. van; ANDRADE, J.C.; CANTARELLA, H.; QUAGGIO, J.A. Análise química para avaliação da fertilidade de solos tropicais. Campinas: Instituto Agronômico, 2001. 285p.

REDDY, A.R.; REDDY, K.R.; HODGES, H.F. Mepiquat chloride (PIX) induced changes in photosynthesis and growth of cotton. Plant Growth Regulation, v.20, p.179-183, 1996.

REDDY, K.R.; BOONE, M.L.; REDDY, A.R.; HODGES, H.F.; TURNER, S.B.; McKINION, J.M. Developing and validating a model for plant growth regulator. Agronomy Journal, v.87, p.11001105, 1995.

REDDY, V.R.; BAKER, D.N.; HODGES, H.F. Temperature and mepiquat chloride on cotton canopy architecture. Agronomy Journal, v.82, p.190-195, 1990.

REDDY, V.R.; TRENT, A.; ACOCK, B. Mepiquat chloride and irrigation versus cotton growth and development. Agronomy Journal, v.84, p.930-933, 1992.

ROSOLEM, C.A.; CALONEGO, J.C.; FOLONI, J.S.S. Lixiviação de potássio da palha de espécies de cobertura de solo de acordo com a quantidade de chuva aplicada. Revista Brasileira de Ciência do Solo, v.27, p.355-362, 2003.

SILVA, M.A. de S. Depósitos da calda de pulverização no solo e em plantas de tiririca (Cyperus rotundus L.) em diferentes condições de aplicação. 2000. 57p. Tese (Doutorado) - Universidade Estadual Paulista, Botucatu.

SOUZA, S.G.; BELTRÃO, N.E.M. Fisiologia. In: BELTRÃO, N.E.M. (Ed.). O agronegócio do algodão no Brasil. Brasília: Embrapa Comunicação para Transferência de Tecnologia, 1999. p.87109.

STUART, B.L.; ISBELL, V.R.; WENDT, C.W.; ABERNATHY, J.R. Modification of cotton relations and growth with mepiquat chloride. Agronomy Journal, v.76, p.651-655, 1984. 